\title{
Article \\ A Proposal of Vertical MOSFET and Electrothermal Analysis for Monolithic 3-D ICs
}

\author{
Jia-He Zhu ${ }^{1}$, Da-Wei Wang ${ }^{1,2, * \mathbb{D}}$, Wen-Sheng Zhao ${ }^{1, *(\mathbb{D}}$, Jia-Yun Dai ${ }^{3}$ and Gaofeng Wang ${ }^{1} \mathbb{D}$ \\ 1 MOE Engineering Research Center of Smart Microsensors and Microsystems, School of Electronics and \\ Information, Hangzhou Dianzi University, Hangzhou 310018, China; zhujiahe0819@126.com (J.-H.Z.); \\ gaofeng@hdu.edu.cn (G.W.) \\ 2 State Key Laboratory of Millimeter Waves, Nanjing 210096, China \\ 3 Science and Technology on Monolithic Integrated Circuits and Modules Laboratory, Nanjing 210016, China; \\ jydai2016@163.com \\ * Correspondence: davidw.zoeq@gmail.com (D.-W.W.); wsh.zhao@gmail.com (W.-S.Z.); \\ Tel.: +86-173-2604-0757 (D.-W.W.); +86-150-8868-7701 (W.-S.Z.)
}

Citation: Zhu, J.-H.; Wang, D.-W.; Zhao, W.-S.; Dai, J.-Y.; Wang, G. A Proposal of Vertical MOSFET and Electrothermal Analysis for Monolithic 3-D ICs. Electronics 2021 10, 2241. https://doi.org/10.3390/ electronics10182241

Academic Editor: Myounggon Kang

Received: 27 August 2021

Accepted: 9 September 2021

Published: 12 September 2021

Publisher's Note: MDPI stays neutral with regard to jurisdictional claims in published maps and institutional affiliations.

Copyright: (c) 2021 by the authors. Licensee MDPI, Basel, Switzerland. This article is an open access article distributed under the terms and conditions of the Creative Commons Attribution (CC BY) license (https:// creativecommons.org/licenses/by/ $4.0 /)$.

\begin{abstract}
In this paper, an innovative vertical MOSFET based on through-oxide via (TOV) technology is proposed for silicon-on-insulator (SOI)-based monolithic 3-D ICs. The proposed vertical MOSFET is investigated numerically. It was found that SOI can effectively reduce the parasitic capacitance, leakage current, power consumption, as well as suppress the pulse current interference of the substrate. The simulated results indicate that the proposed MOSFET possesses excellent characteristics in saturation current over $1500 \mu \mathrm{A}$, sub-threshold swing of $69 \mathrm{mV} / \mathrm{dec}$, and ON/OFF current ratio of $1.28 \times 10^{11}$. Moreover, as temperature is a critical factor for the performance degradation of semiconductor devices, electrothermal simulations are conducted to predict the influence of the self-heating effect on device characteristics. The results show that device characteristics slightly deteriorate, but can still acceptable in their applications.
\end{abstract}

Keywords: vertical MOSFET; through-oxide-via; silicon-on-insulator; electrothermal simulation; self-heating effect

\section{Introduction}

Given the ever-increasing requirement for high-density and high-performance integrated circuits (IC), semiconductor processes has evolved along with Moore's law constantly for more than half a century [1]. However, with the process approaching the physical limit of the material, various undesirable problems, such as short channel effect emerge in planar MOSFETs, and thereby hinder the IC development [2]. In order to address with these issues, monolithic three-dimensional (M3D) IC, which possesses excellent performance and power efficiency, has been proposed and is now rapidly developing [3]. M3D ICs are implemented in silicon-on-insulator (SOI) technology, and through-oxide vias (TOV) are patterned through the buried oxide (BOX) to offer electrical connectivity between tiers [4,5].

In addition to the improvements in integration technology, significant efforts have been devoted to the optimization of transistors. In the past several years, vertical MOSFETs have attracted much attention and been extensively studied, due to their advantages in occupying small areas and electrostatic control ability [6,7]. In [8], a Si nanowire-based tunneling field-effect transistor (TFET), with vertical gate-all-around (GAA) structure was proposed. A very low room-temperature (RT) subthreshold swing (SS) was achieved. The low SS of TFET arises from the silicidation of the nanowire, which can activate the sourceside dopants to achieve a steep dopant profile. However, the diameter nonuniformity along the nanowire length results in asymmetry in the device's subthreshold characteristic. To solve this issue, an improved InGaAs dry etch technology was reported in [9], and 
the subthreshold asymmetry improved to some extent. Based on conventional FinFET, Zheng et al. reported on an improved device architecture, which was named as iFinFET [10]. It was demonstrated that iFinFET exhibits better electrostatic integrity and scalability than FinFET, as well as less gate capacitance than GAA FET. In [11], a vertical InAs MOFET was fabricated on $\mathrm{Si}$ substrate for low power and high frequency applications, but its large parasitic capacitance, induced by metal overlap, limits its performance. Some impressive high power vertical transistors were reported. In [12], a vertical power MOSFET with high- $\mathrm{k}$ gate insulator and hexagonal structure was designed, and exhibited a low onresistance but slow response speed. In [13], a large-area in-situ oxide, GaN interlayer-based vertical trench MOSFET was presented, with extensive potential for its application in power electronics.

Considering their various advantages, it has been intuitive to apply vertical MOSFETs in M3D ICs. The transistors in M3D are implemented with SOI technology, which could effectively reduce the parasitic capacitance, leakage current, and power consumption [14-16]. While, the reported research on SOI transistors mainly focused on planar devices [17-19], there has rarely been any reports on SOI-based vertical MOSFET; the motivation for our present research. On the other hand, it is worth noting that thermal issues are critical in M3D ICs due to the stacking of power devices, and high temperatures can cause the degradation of device performance [20]. Therefore, special attention should be given to the self-heating effect (SHE) of transistors. Electrothermal simulation is an effective way to predict device characteristics and various tools have been developed to conduct numerical analyses [21,22]. Among these tools, COMSOL Multiphysics software is capable of modeling and simulating the electrothermal process of semiconductor devices [23-25], and thus has been adopted in this study.

The rest of this paper is arranged as follows. The design and geometry of the proposed SOI-based vertical MOSFET, as well as a practical fabrication scheme are detailed in Section 2. The potential applications for the proposed device are briefly described. In Section 3, numerical simulation schemes for the electrical and electrothermal coupling simulations of the proposed device are presented. Then, the characteristics of the proposed vertical MOSFET are numerically simulated by following the designed schemes, and reliable results are achieved to evaluate the performance of the proposed device in Section 4. Some conclusions are finally drawn in Section 5.

\section{Modeling}

\subsection{Device Model}

Figure 1 shows the geometry of the proposed vertical MOSFET. A thin $\mathrm{SiO}_{2}$ layer is formed on top of the Si wafer for isolation, with the source electrode and interconnects processed on it. The doped Si film bonds on the dioxide layer works as the active layer for transistor fabrication. Contrary to the conventional planar SOI-based MOSFET, the heavily doped drain and source regions are located at the top and bottom surface of the proposed device, respectively. TOV is processed to construct the gate electrode. In particular, the hydrogen silsesquioxane layer is formed to determine the position of the gate electrode, and the insulator layers are fabricated on top of the active Si film for the electrodes and layout.

The proposed vertical MOSFET is potential in the implementation of M3D ICs. For example, the low on-resistance of vertical transistor can work as switch to control the electrical connection between tiers, and a 3-D inverter could be constructed in M3D ICs, as shown in Figure 2. The drains of PMOS and NMOS are connected by conductor trace line as the output, and their gates act as the input. 


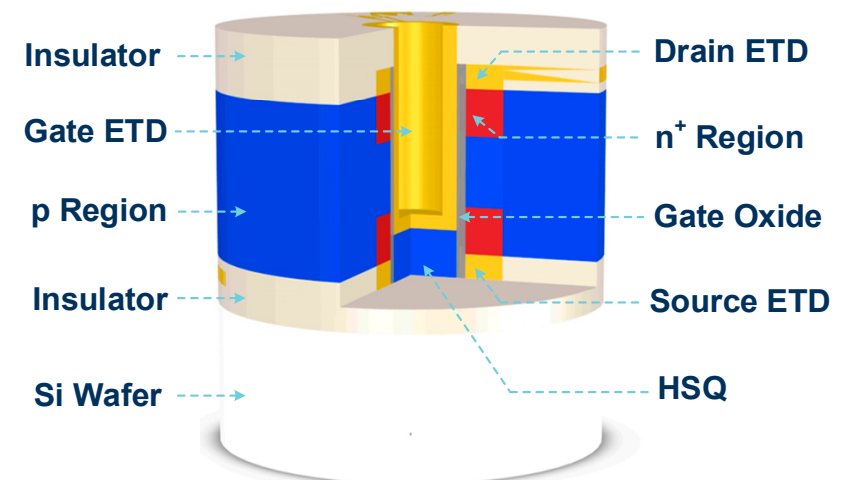

Figure 1. Schematic of the proposed vertical MOSFET.

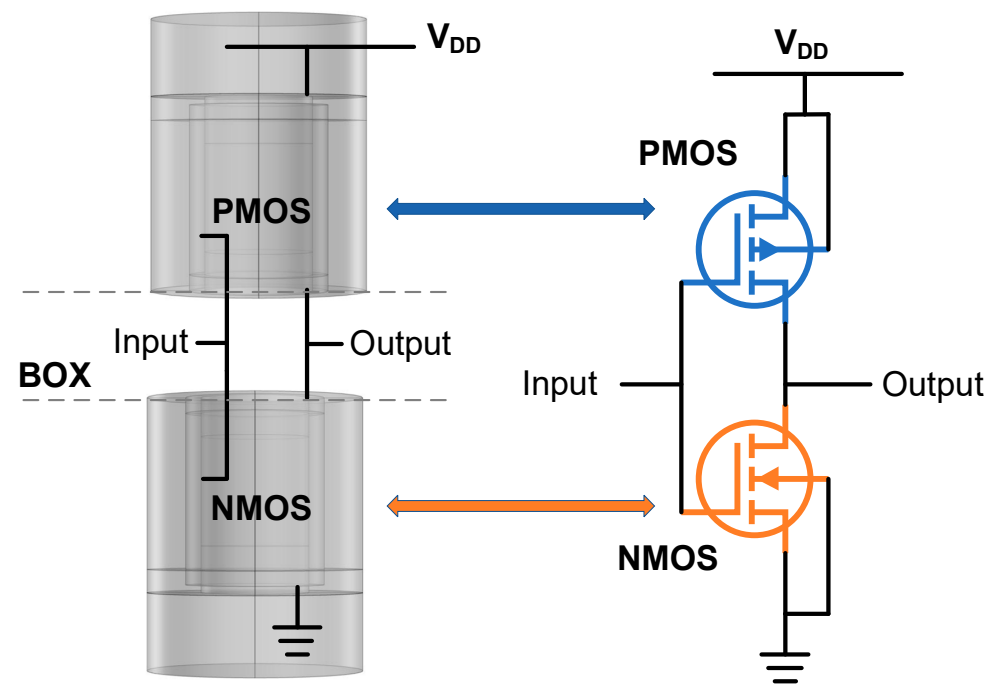

Figure 2. Schematic of 3-D inverter constructed by the proposed vertical MOSFETs for M3D ICs.

\subsection{Fabrication Process}

Here, a possible fabrication process is described in detail for the proposed vertical MOSFET to illustrate the feasibility of the proposed design scheme. As shown in Figure 3, the proposed transistor can be fabricated in two stages. In the first stage, a $\mathrm{SiO}_{2}$ is formed on the top of the Si wafer through thermal oxidation to serve as BOX, and the source electrode is deposited. In the second stage, a $p$-doped Si wafer is pre-treated by piranha $\left(98 \% \mathrm{H}_{2} \mathrm{SO}_{4}: 30 \% \mathrm{H}_{2} \mathrm{O}_{2}=3: 1\right)$ and DI water first [26], and then impurity doping and silicon direct bonding are conducted. During this process, the device region on the bonded surface is firstly doped by ion implantation method and then the Si film is boned onto $\mathrm{Si} / \mathrm{SiO}_{2}$ substrate by direct bonding technology. Following is the doping on the other side of SOI is conducted and a rapid thermal anneal (RTA) is carried out in $\mathrm{N}_{2}$ ambient to activate $n$-doping ions and restore crystal lattices damage. Next, a hole is etched with the assistance of a photoresist mask. An improved Bosch is employed for hole etching, followed by several steps to remove $\mathrm{CF}_{\mathrm{x}}$ polymers in the inside of the hole $[27,28] . \mathrm{A} \mathrm{SiO}_{2}$ layer is grown by thermal oxidation on the top of wafer, followed by a thermal atomic layer deposition (thALD) of $\mathrm{Al}_{2} \mathrm{O}_{3}$. After that, the drain electrode is deposited on the top of $\mathrm{n}^{+}$-doped region. At last, a HSQ spacer layer is processed [29], and a layer of metal film is sputtered on the spacer to form the gate electrode. So far, the proposed vertical transistor is fabricated. The 3-D CMOS inverter, shown in Figure 2, can effectively reduce the interconnect length, based on the direct interconnection between PMOS and NMOS through the hole via. 


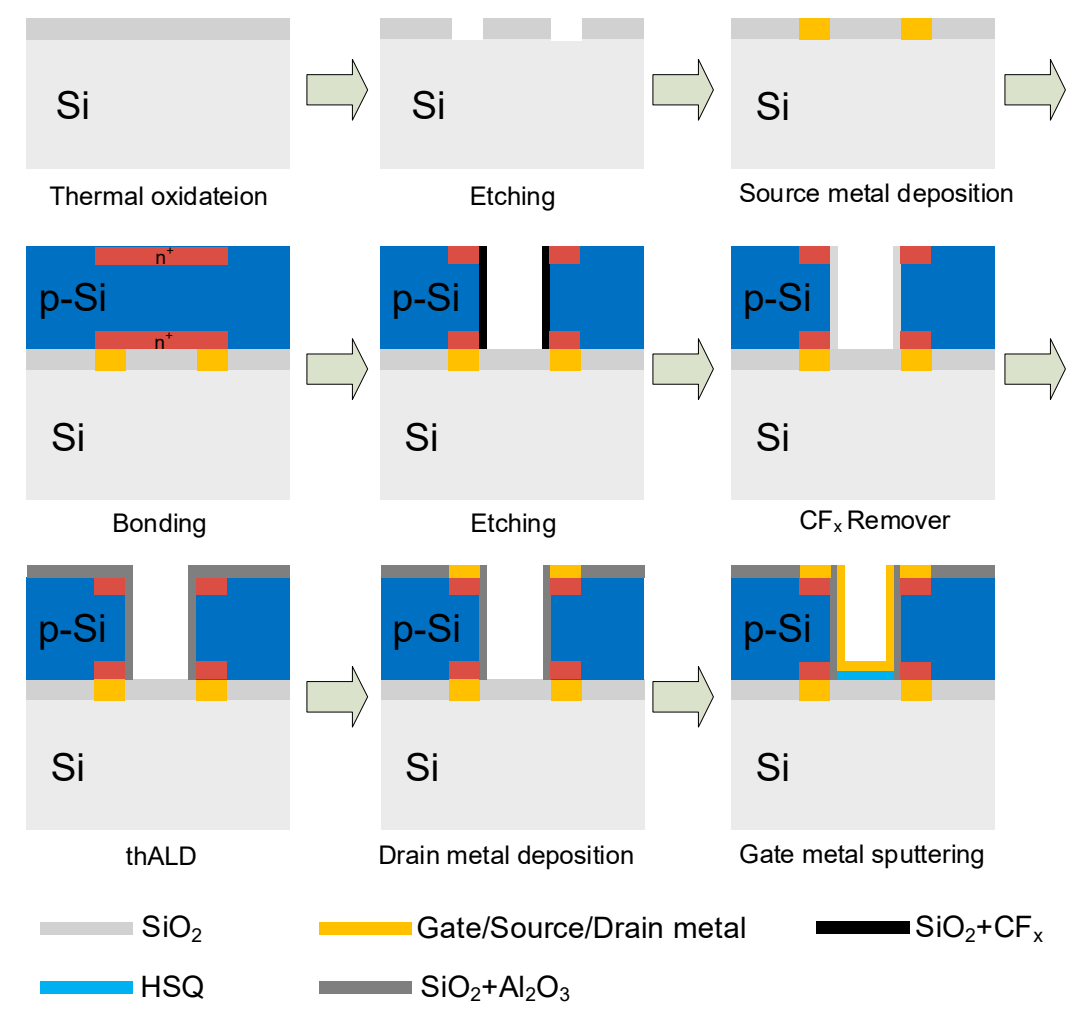

Figure 3. Flowchart of the fabrication process for the proposed vertical transistor.

\section{Simulation}

In this section, the electrical characteristics of the proposed vertical transistor, including SS, $I_{\text {on }} / I_{\text {off }}$ ratio, threshold voltage and drain current in the conduction state are studied using the COMSOL Multiphysics software. At the same time, the electrothermal coupling simulation is also conducted to estimate the degradation in device performance as SHE is a significant concern about device performance and reliability [30]. In the simulations, the drift-diffusion model, composed of carrier current continuity equations and Poisson equation, is employed to characterize the electrical property, which reads:

$$
\begin{gathered}
\nabla \cdot \vec{J}_{n}=\frac{\partial n}{\partial t}+R_{n}+G \\
-\nabla \cdot \vec{J}_{p}=\frac{\partial p}{\partial t}+R_{p}+G \\
\nabla^{2} \varphi=\frac{q}{\varepsilon}\left(n-p+N_{A}^{-}-N_{D}^{+}\right)
\end{gathered}
$$

where $n$ is electron density, $p$ is hole density, $\varphi$ is the potential, $q$ is the unit charge, $R_{n}$ and $R_{p}$ represent the net recombination rate, $N_{\mathrm{D}}^{+}$and $N_{\mathrm{A}}^{-}$represent the concentration of ionized donor and acceptor, $\vec{J}_{n}$ and $\vec{J}_{p}$ are the electron and hole current density, which can be written as:

$$
\begin{aligned}
& J_{n}=q n \mu_{n} \nabla E_{c}+\mu_{n} k_{B} T \nabla n+q n D_{n, t h} \nabla \ln (T) \\
& J_{p}=q p \mu_{p} \nabla E_{v}-\mu_{p} k_{B} T \nabla p-q p D_{p, t h} \nabla \ln (T)
\end{aligned}
$$

where $k_{B}$ is the Boltzmann constant, $T$ is the temperature, $E_{c}$ and $E_{v}$ are the conduction band and valence band, $D_{n, t h}$ and $D_{p, t h}$ are carrier diffusivities, and $\mu_{n}$ and $\mu_{p}$ are the electron mobility and hole mobility, respectively. In the semiconductor module, the finite vol approach, combined with Scharfetter-Gummel current model is utilized to implement the numerical calculation of carrier current continuity equations. 
To implement the thermal simulation, the Fourier heat transfer equation is numerically calculated by finite element method in the solid heat transfer module. The heat transfer equation can be written as:

$$
\rho C_{P} u \cdot \nabla T+\nabla \cdot(-k \nabla T)=Q
$$

where $\rho$ is the material density, $C_{P}$ is the heat capacity, $u$ is the translatory velocity, $k$ is the thermal conductivity and $Q$ is the unit volume heat source which provided by the semiconductor module. To verify the accuracy of simulation process, a nanowire MOSFET, shown in Figure 4a, with $20 \mathrm{~nm}$ channel length, 1nm gate oxide thickness, $5 \mathrm{~nm}$ nanowire radius is simulated. In Figure $4 b$, the simulated results are compared with experimental data reported in [31], and good agreement is achieved.

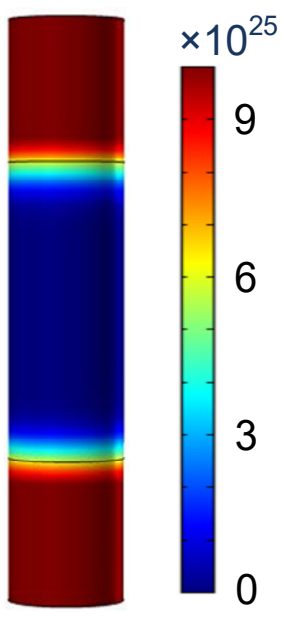

(a)

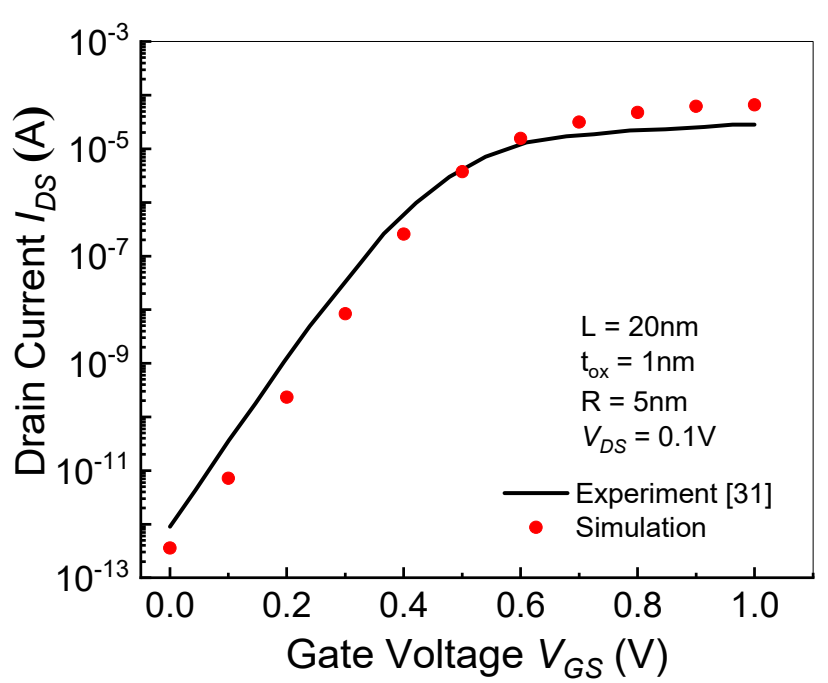

(b)

Figure 4. (a) The structure and doping distribution of nanowire MOSFET; (b) comparison of output characteristics of nanowire MOSFET with published results.

\subsection{Electrical Performance}

To simulate the characteristics of the proposed vertical transistor, a geometric model is established in COMSOL with $3 \mu \mathrm{m}$ Si film thickness, $300 \mathrm{~nm}$ TOV radius, $1.5 \mu \mathrm{m}$ gate width, and $30 \mathrm{~nm}$ gate oxide layer thickness. Then, an analytical doping physical model is employed to achieve doping distribution in the active region of the vertical transistor. Implantations of donor with a peak of $1 \times 10^{20} \mathrm{~cm}^{-3}$ and a $0.15 \mu \mathrm{m}$ depth Gaussian distribution junction are made over a $p$-type background with a constant acceptor profile of $1 \times 10^{16} \mathrm{~cm}^{-3}$ magnitude to form source and drain regions. The detail doping information is illustrated in Figure 5, where Figure 5a presents the $n$-type ion doping diagram, and Figure $5 \mathrm{~b}$ displays donor ion concentration $N_{d}$ and acceptor ion concentration $N_{a}$ as a function of doping depth.

In order to feature the physical process in a transistor, the classical drift-diffusion model is applied. In the simulations, the metal-semiconductor contacts of the source and drain are set to be ideal ohmic contacts, while Schottky contact is assigned to the gate. Moreover, the Shockley-Read-Hall model is adopted to describe the recombinationgeneration occurring in the active region. The analysis of electrical performance mainly focuses on transfer curve and output curve. During the simulations, the result of the transfer curve is utilized as the initial value for the output curve calculation. 


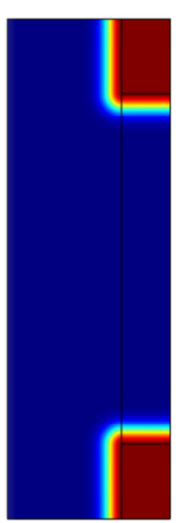

(a)

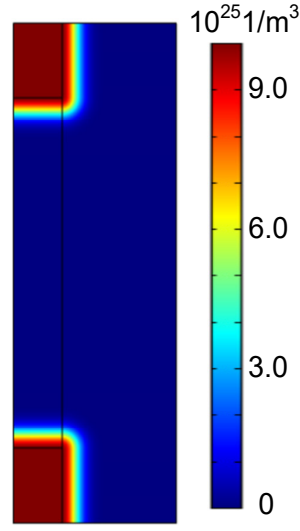

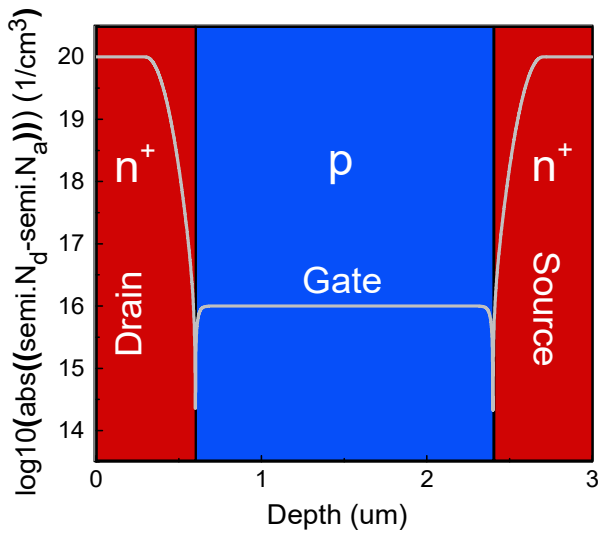

(b)

Figure 5. (a) The section of n-type ion doping; (b) distribution curve of $N_{d}$ and $N_{a}$ with respect to doping depth.

\subsection{Electrothermal Coupling Simulation}

As mentioned above, SHE is a main cause for the performance degradation, as SHE can induce a temperature rise in the transistors. The temperature rise in transistor could result in the reduction of electron/hole mobility, thereby increasing the on-resistance and affecting the transistor performance. Therefore, the electrothermal coupling simulation should be conducted to estimate the influence of SHE on the transistor performance.

Compared to the electrical simulation, the electrothermal coupling process is described by a mathematical equation system composed of drift-diffusion equation, thermal conduction equation, and coupling equations. The coupling equations work as the bridge linking the electric and thermal fields. In COMSOL software, two modules, i.e., Semiconductor and Heat Transfer in Solids, are utilized to realize the electrothermal coupling simulation of transistors. The semiconductor heat source and temperature-dependent parameters are utilized as the direct and indirect coupling equations. In the simulations, the initial temperature in the calculation region is set to be RT, i.e., $293.15 \mathrm{~K}$. The electrical equations are firstly solved utilizing the Semiconductor module, and with the heat source achieved by electrical simulation as an input, the thermal conduction equation is subsequently solved to update the temperature distribution. Based on the calculated temperature, the temperature-dependent parameters are updated and arranged for the next calculation loop. After the coupling simulation, the output curve affected by SHE can be measured, and by comparing it with the curve achieved by pure electrical simulation, the influence of SHE can be observed intuitively.

\section{Results and Discussion}

Following the modeling and simulation schemes described above, the electrical and electrothermal characteristics of the proposed vertical MOSFET are captured.

The electrical simulations of the proposed transistor are carried out first. In Figure 6, the threshold voltages (Vth), SS, Ion/Ioff ratio curves versus gate biasing voltage are presented. The transfer curves with drain voltage of $0.01 \mathrm{~V}, 0.1 \mathrm{~V}$ and $2 \mathrm{~V}$ are presented in Figure $6 \mathrm{a}$, and a $0.45 \mathrm{~V}$ threshold voltage is observed. This low threshold voltage is mainly due to the low background doping. In Figure $6 \mathrm{~b}$, the $I_{D S}-V_{G S}$ characteristic curves of the vertical transistor are presented in log-scale, and it can be observed that the proposed vertical transistor exhibits a high $I_{\text {on }} / I_{\text {off }}$ ratio and low SS. For example, the $I_{\text {on }} / I_{\text {off }}$ ratio for $V_{D S}=0.1 \mathrm{~V}$ could reach as high as $9.6 \times 10^{9}$ and a low SS of $69 \mathrm{mV} / \mathrm{dec}$ is achieved for case of $V_{D S}=0.01 \mathrm{~V}$. The SS depends on minority carrier, i.e., the subthreshold current of MOSFET is the minority carrier diffusion current [32]. Accordingly, the SS is related to the factors that affect the minority carrier injection efficiency and its movement, and the factors affecting the gate control ability, include substrate doping concentration, temperature, 
and so on. Another index demonstrating an excellent gate controllability in the proposed transistor is the low drain induced barrier lowering (DIBL), which is less than $20 \mathrm{mV}$ between cases of $V_{D S}=0.1 \mathrm{~V}$ and $2 \mathrm{~V}$.

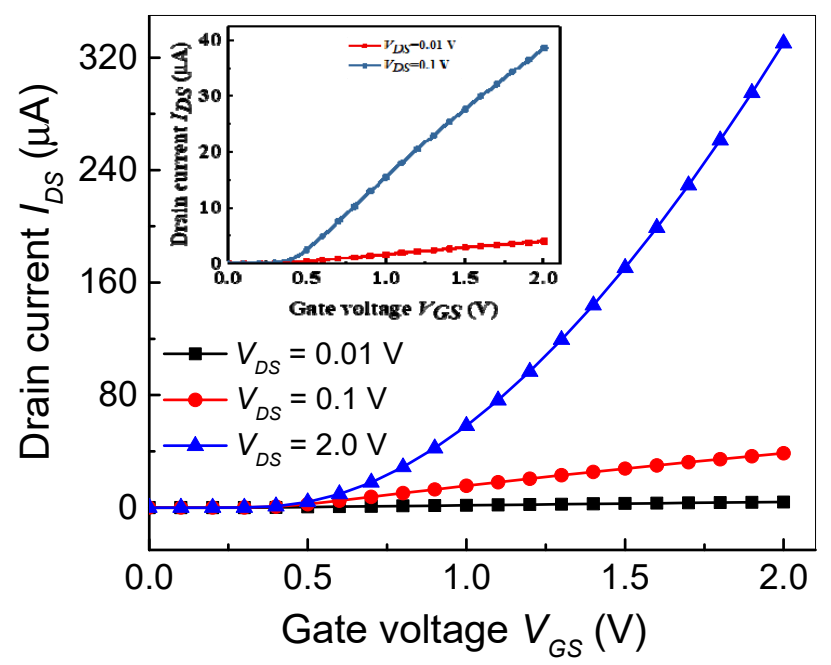

(a)

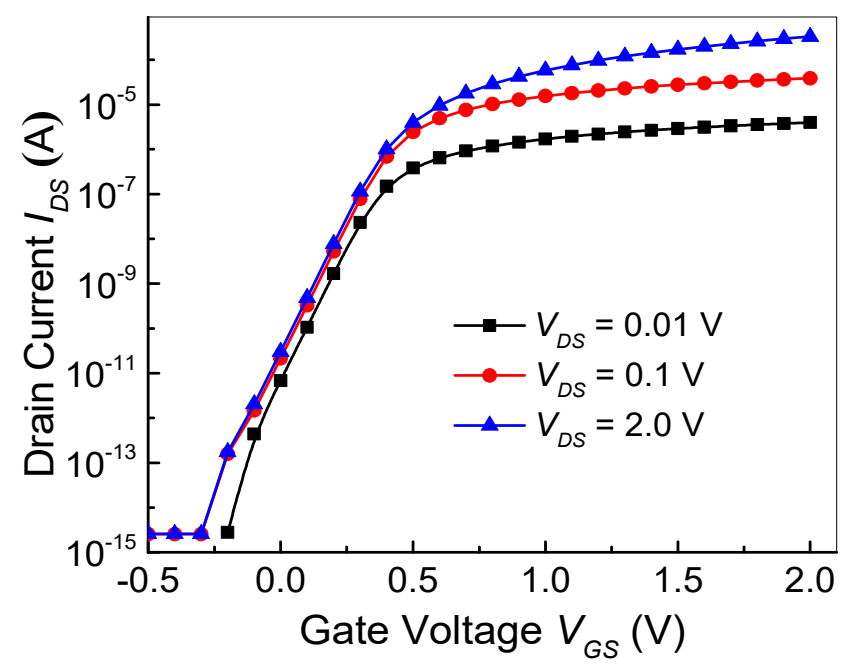

(b)

Figure 6. (a) $I_{D S}-V_{G S}$ characteristic of the proposed vertical MOSFET; (b) transfer characteristic curve of the proposed vertical MOSFET.

The electrical characteristics of the proposed vertical transistor are summarized in Table 1. It can be concluded that the proposed transistor possesses excellent switching performance with a low SS $(69 \mathrm{mV} / \mathrm{dec})$, which approaches the limit value of $60 \mathrm{mV} / \mathrm{dec}$, indicating that the proposed vertical MOSFET is qualified to be a switch. In addition, the high $I_{o n} / I_{\text {off }}$ ratio exhibits excellent gate controllability, owing to vertical channel blocking any parasitic leakage path [33]. The output characteristic curves of the vertical MOSFET with changing $V_{G S}$ are shown in Figure 7a, where a maximum drain saturation current of $1500 \mu \mathrm{A}$ is obtained in case of $V_{G S}=4 \mathrm{~V}$. Moreover, the 3-D electron concentration distribution of the on-state is illustrated in Figure $7 \mathrm{~b}$, where the formation of vertical current channel can be clearly observed.

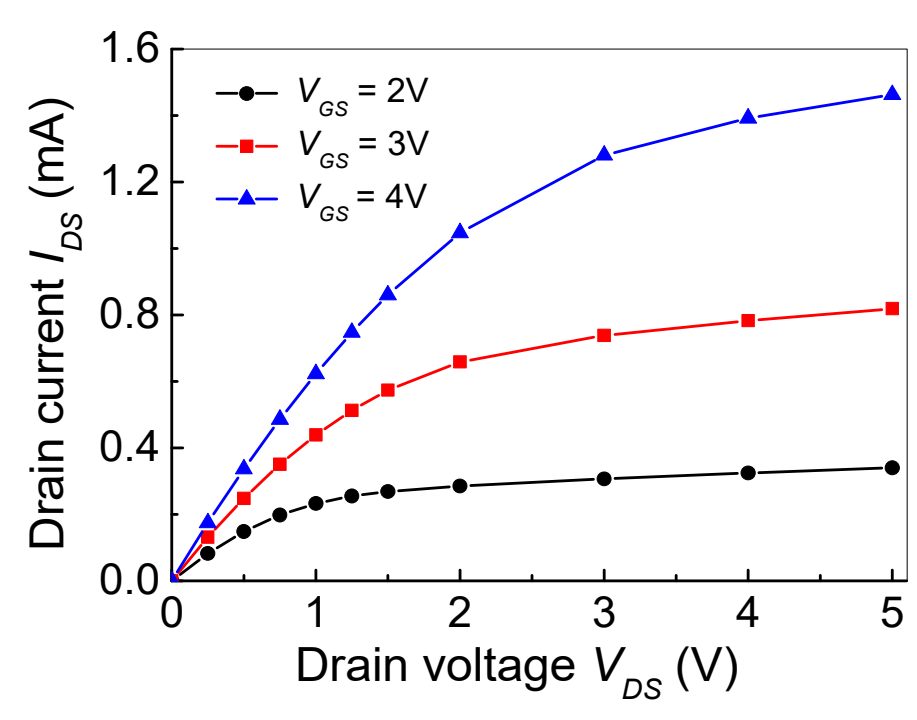

(a)

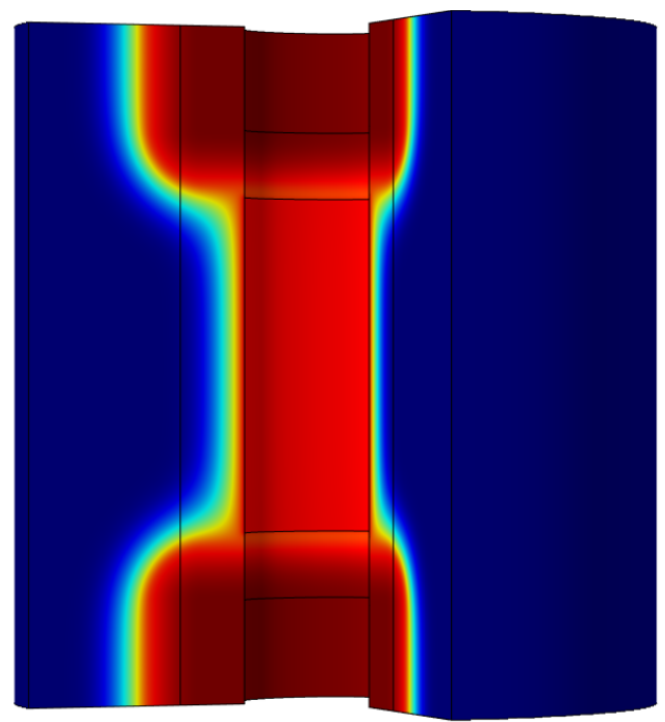

(b)

Figure 7. (a) Output curves of vertical MOSFET; (b) channel of vertical MOSFET in on-state. 
Table 1. Electrical parameters of the Vertical MOSFET.

\begin{tabular}{ccc}
\hline$V_{\boldsymbol{D S}}(\mathrm{V})$ & $\boldsymbol{I}_{\text {on }} / \boldsymbol{I}_{\text {off }}$ Ratio & SS (mV/dec) \\
\hline 0.01 & $1.4 \times 10^{9}$ & 69 \\
0.1 & $1.52 \times 10^{10}$ & 79 \\
2 & $1.28 \times 10^{11}$ & 81 \\
\hline
\end{tabular}

The performance indexes of the proposed vertical transistor are compared with previous reported works in Table 2. The improvement in the $I_{o n} / I_{\text {off }}$ ratio is achieved from the gate electrode surrounding the whole channel region which enhances the gate controllability, and the low SS due to the low substrate doping concentration and outstanding gate controllability.

Table 2. Comparison of electrical performance.

\begin{tabular}{ccccc}
\hline Ref. & $\boldsymbol{V}_{\boldsymbol{t h}} \mathbf{( V )}$ & $\boldsymbol{I}_{\boldsymbol{o n}} / \boldsymbol{I}_{\boldsymbol{o f f}}$ Ratio & $\mathbf{S S}(\mathbf{m V} / \mathbf{d e c})$ & $\boldsymbol{I}_{\boldsymbol{D S}}(\boldsymbol{\mu A})$ \\
\hline$[6]$ & 0.29 & $1.4 \times 10^{3}$ & 90 & - \\
{$[28]$} & -0.63 & $1.2 \times 10^{4}$ & - & 430 \\
{$[34]$} & 0.79 & $1 \times 10^{8}$ & 62 & 5.3 \\
{$[35]$} & 4.1 & $2 \times 10^{10}$ & - & 180 \\
This work & 0.45 & $1.28 \times 10^{11}$ & 69 & 1500 \\
\hline
\end{tabular}

In practice, however, the SHE in active region could induce temperature rise, and then result in performance degradation. Consequently, the electrothermal coupling simulations are conducted to evaluate the influence of SHE on the transistor performance. The obtained temperature distributions on the cross-section along the axis direction for different biasing configurations are presented in Figure 8. A maximum temperature of $410 \mathrm{~K}$ is observed in case of $V_{D S}=5 \mathrm{~V}$ and $V_{G S}=4 \mathrm{~V}$, as the channel current is larger than in other three cases, as shown in Figure 7. Generally, there is no significant increase in the device temperature, which is mainly due to the satisfactory heat dissipation performance of TOV [36].

It can be observed that a high temperature region is located at the current channel near the drain side, and the heat gradually propagates outward to form temperature gradient. As the drain voltage increases, the distribution of high temperature region becomes more concentrated. This is mainly because the current channel near the drain region is the narrowest, which results in the largest current density, i.e., the maximum power density. To intuitively observe this phenomenon, the distributions of the generated heat power with $V_{D S}=3 \mathrm{~V}, V_{G S}=4 \mathrm{~V}$ and $V_{D S}=5 \mathrm{~V}, V_{G S}=4 \mathrm{~V}$ are shown in Figure 9.

During the transistor operation, the internal joule heat accumulates and induces the temperature rise. The excessive temperature rise could degrade the device performance and affect the reliability [37]. The output characteristics of the proposed vertical MOSFET, based on the electrothermal coupling simulations, are obtained and illustrated in Figure 10. It is evident that the drain currents obtained by electrothermal coupling simulation are slightly smaller than those obtained by electrical simulations. Compared with high $V_{G S}$ case, the difference between $I_{D S}$, achieved by electrical and electrothermal simulations, is smaller than in low $V_{G S}$. This is mainly because a lower channel current corresponds to lower temperature rise, i.e., lower performance degradation. On the other hand, for the same $V_{G S}$, the current drop increases with $V_{D S}$. Similarly, this arises from the device temperature increases with $V_{G S}$. As the device current level drops slightly, it can be concluded that the proposed transistor possesses good thermal stability. 


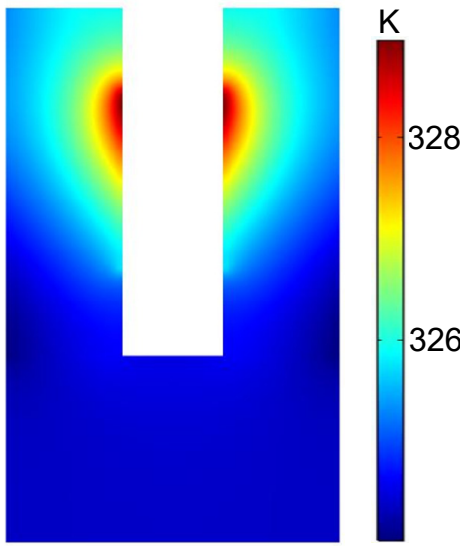

(a)

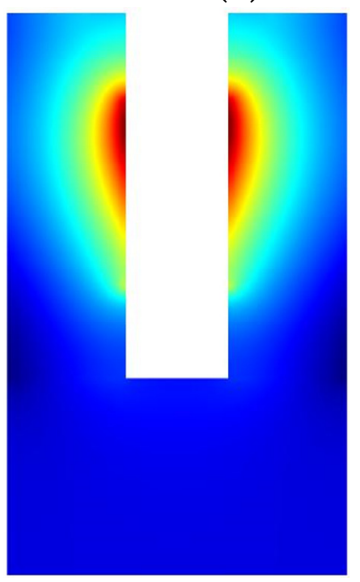

(c)

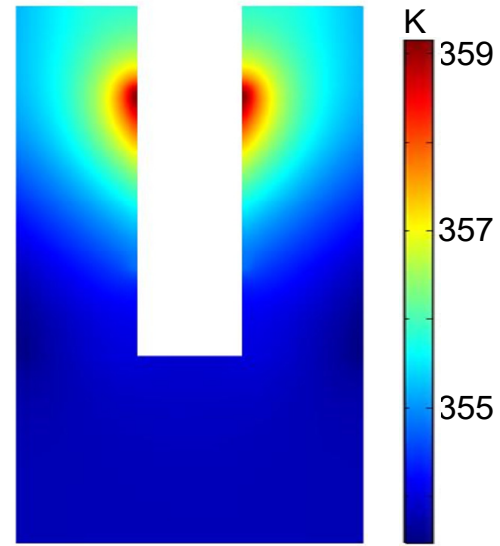

(b)

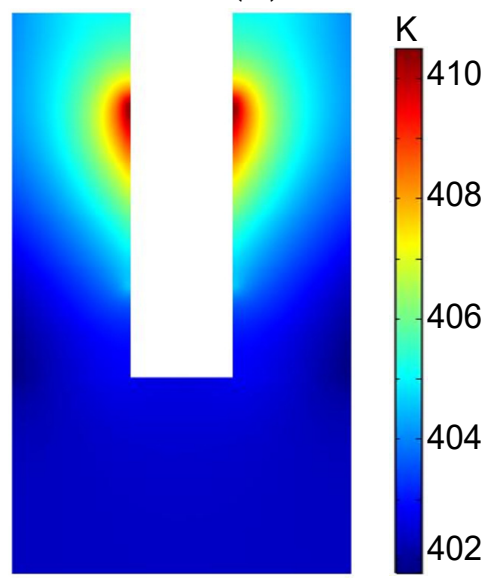

(d)

Figure 8. Temperature profile of the vertical MOSFET in the working state. (a) $V_{D S}=3 \mathrm{~V}, V_{G S}=3 \mathrm{~V}$, (b) $V_{D S}=5 \mathrm{~V}, V_{G S}=3 \mathrm{~V}$, (c) $V_{D S}=3 \mathrm{~V}, V_{G S}=4 \mathrm{~V}$, (d) $V_{D S}=5 \mathrm{~V}, V_{G S}=4 \mathrm{~V}$.

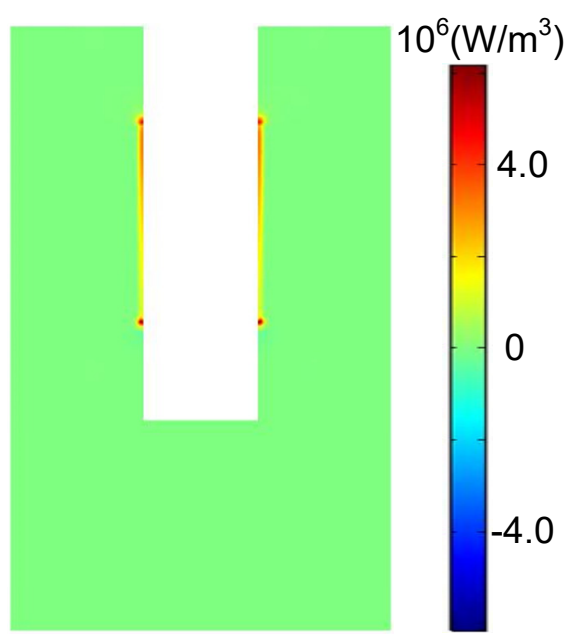

(a)

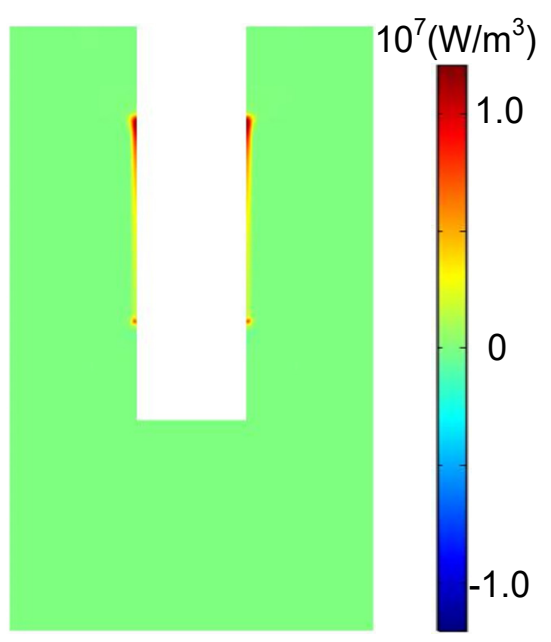

(b)

Figure 9. Distributions of power generated in the on-state device: (a) $V_{D S}=3 \mathrm{~V}, V_{G S}=4 \mathrm{~V}$; (b) $V_{D S}=5 \mathrm{~V}, V_{G S}=4 \mathrm{~V}$. 


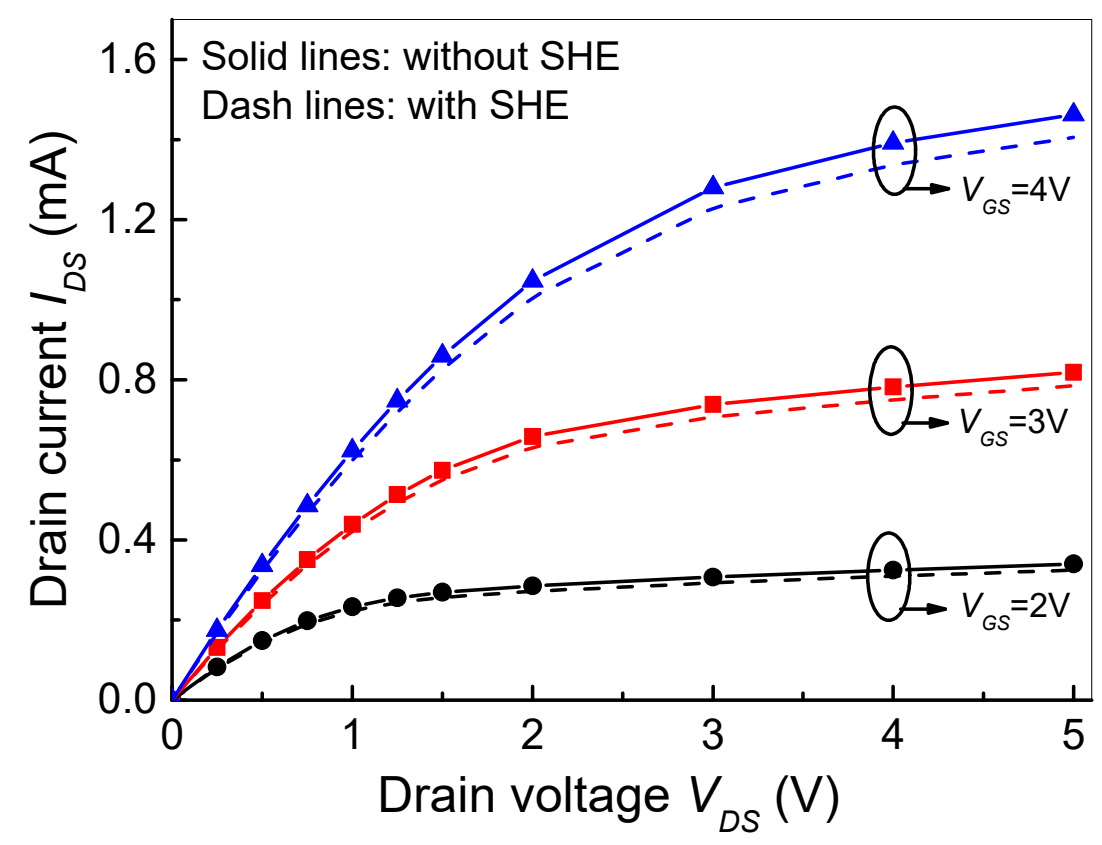

Figure 10. Output characteristic curve of vertical MOSFETs with, and without, consideration of the SHE.

Further, the influences of geometric parameters on the performance of the proposed vertical MOSFET are investigated, with some results illustrated in Figure 11. It can be observed that the saturation current of the MOSFET is improved with decreasing channel length $(L)$ and gate oxide thickness $\left(t_{o x}\right)$, and with the increasing relative permittivity of gate oxide $\left(\varepsilon_{\text {gate }}\right)$. However, the increase in drain current could result in larger power consumption and then aggravate SHE. The electrothermal coupling simulations have been carried out, and the maximum temperatures in MOSFETs are presented in Figure 12. Consistent with the saturation current, the maximum temperature in MOSFETs increases with the decrease in channel length and gate oxide thickness. It can also be observed that the maximum temperature in MOSFET with $L=1200 \mathrm{~nm}, t_{0 x}=30 \mathrm{~nm}$, and $\varepsilon_{\text {gate }}=20$ is lower than those in MOSFETs with $L=600 \mathrm{~nm}, t_{o x}=30 \mathrm{~nm}$, and $\varepsilon_{\text {gate }}=4.5$, and with $L=1200 \mathrm{~nm}, t_{o x}=20 \mathrm{~nm}$, and $\varepsilon_{\text {gate }}=4.5$, although it possesses largest saturation current. This is mainly due to the high thermal conductivity of $\mathrm{HfO}_{2}$. Although the decreasing gate oxide thickness could increase the saturation current to some extent, the SHE would be aggravated, and the probability for reliability issues such as electric field breakdown, would arise [38]. 


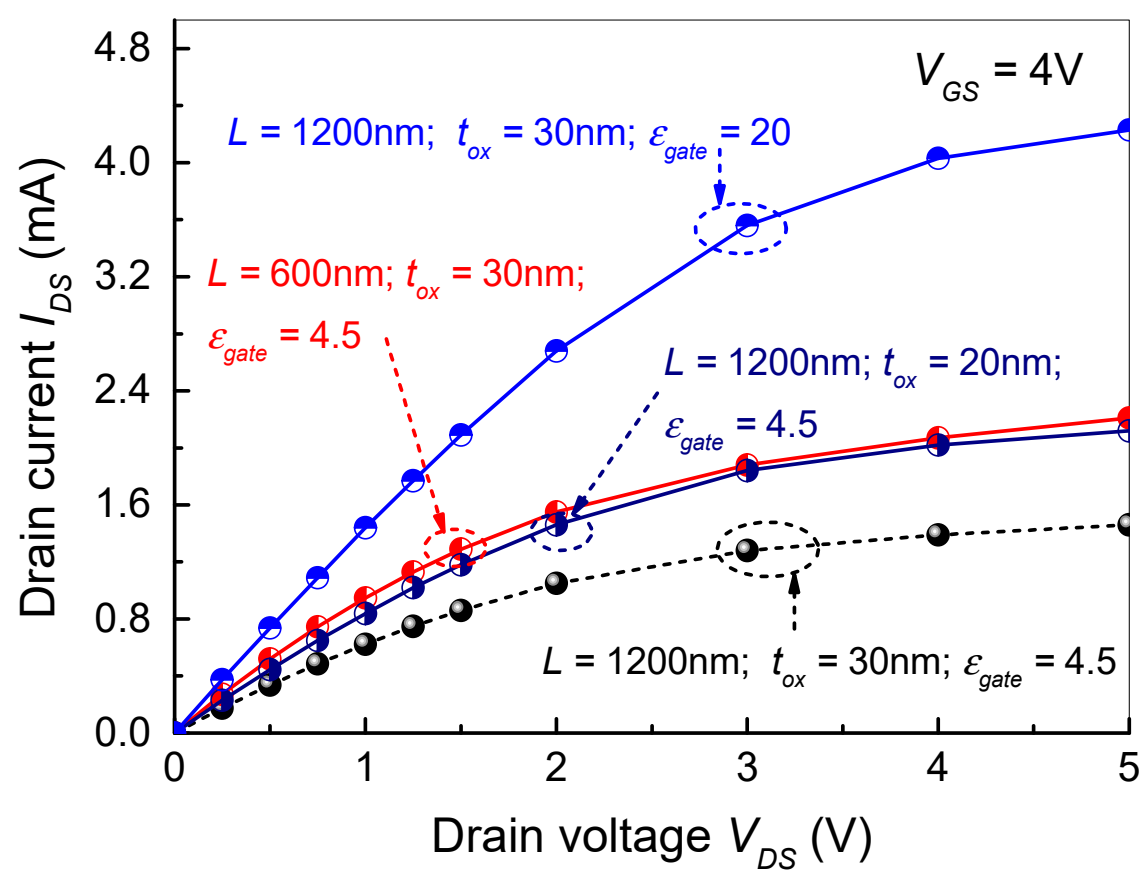

Figure 11. Drain currents as functions of drain voltages for different parameter configurations.

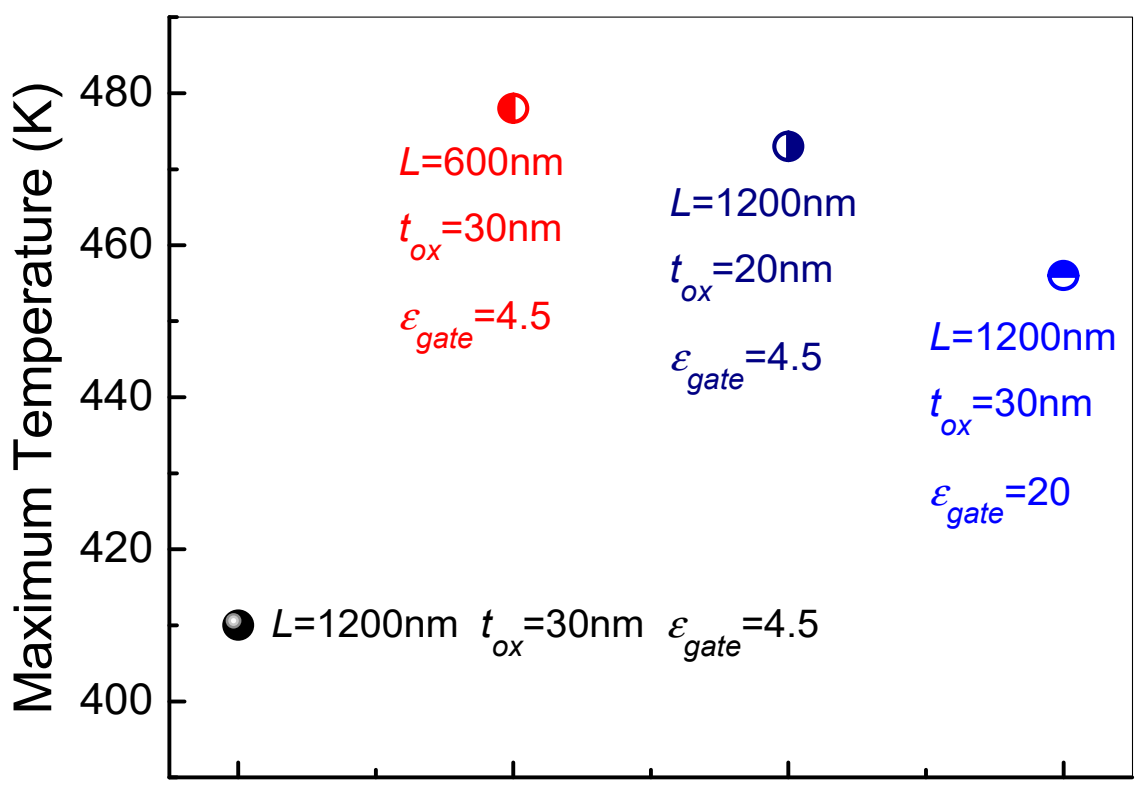

Figure 12. Maximum temperatures in vertical MOSFET with different parameter configurations.

\section{Conclusions}

A vertical MOSFET, based on silicon-on-insulator technologies was proposed, and its characteristics were investigated through numerical simulations. An electrical simulation was firstly carried out, and the results indicated that the proposed transistor possesses excellent electrical performance. A $1500 \mu \mathrm{A}$ on-state current $\left(I_{D S}\right)$ was achieved in case of $V_{G S}=4 \mathrm{~V}$ and $V_{D S}=5 \mathrm{~V}$, and excellent current control ability was demonstrated as a $1.28 \times 10^{11} I_{\text {on }} / I_{\text {off }}$ ratio was obtained. Moreover, an extremely low subthreshold swing of $69 \mathrm{mV} / \mathrm{dec}$ was obtained, which makes the proposed MOSFET a useful switch in monolithic 3-D ICs. Further, the electrothermal coupling simulations were conducted to evaluate the influence of self-heating effect on the transistor performance. The results illustrated that the self-heating effect induced a temperature rise in the channel, and then reduced performance. 
Nonetheless, benefiting from the outstanding heat dissipation performance of the central via, the proposed vertical MOSFET exhibited good thermal stability. The proposed vertical MOSFET has potential applications in monolithic 3-D ICs.

Author Contributions: Methodology, writing-original draft preparation, J.-H.Z.; supervision, writingreviewing and editing, D.-W.W.; conceptualization, investigation, W.-S.Z.; data curation, validation, J.-Y.D.; supervision, investigation, G.W. All authors have read and agreed to the published version of the manuscript.

Funding: This research was funded by the National Natural Science Foundation of China, grant number 61934006, 62101170, and 61874038, and the Open Research Fund of State Key Lab of Millimeter Waves, Southeast University, grant number K202231.

Informed Consent Statement: Informed consent was obtained from all subjects involved in the study.

Conflicts of Interest: The authors declare no conflict of interest regarding the publication of this article.

\section{References}

1. Fleetwood, D.M. Evolution of total ionizing dose effects in MOS devices with Moore's law scaling. IEEE Trans. Nucl. Sci. 2018, 65, 1465-1481. [CrossRef]

2. Pourghaderi, M.A.; Pham, A.-T.; Ilatikhameneh, H.; Kim, J.; Park, H.-H.; Jin, S.; Chung, W.-Y.; Choi, W.; Maeda, S.; Lee, K.-H. Universality of short-channel effects on ultrascaled MOSFET performance. IEEE Electron Device Lett. 2018, 39, 168-171. [CrossRef]

3. Hu, X.; Stow, D.; Xie, Y. Die stacking is happening. IEEE Micro 2018, 38, 22-28. [CrossRef]

4. Guler, A.; Jha, N.K. McPAT-Monolithic: An area/power/timing architecture modeling framework for 3-D hybrid monolithic multicore systems. IEEE Trans. Very Large Scale Integr. (VLSI) Syst. 2020, 28, 2146-2156. [CrossRef]

5. Lee, Y.; Lim, S.K. Ultrahigh density logic designs using monolithic 3-D integration. IEEE Trans. Comput. Aided Des. Integr. Circuits Syst. 2013, 32, 1892-1905. [CrossRef]

6. Berg, M.; Kilpi, O.-P.; Persson, K.-M.; Svensson, J.; Hellenbrand, M.; Lind, E.; Wernersson, L.-E. Electrical characterization and modeling of gate-last vertical InAs nanowire MOSFETs on Si. IEEE Electron Device Lett. 2016, 37, 966-969. [CrossRef]

7. Donaghy, D.; Hall, S.; de Groot, C.H.; Kunz, D.V.; Ashburn, P. Design of 50-nm vertical MOSFET incorporating a dielectric pocket. IEEE Trans. Electron Devices 2004, 51, 158-161. [CrossRef]

8. Gandhi, R.; Chen, Z.; Singh, N.; Banerjee, K.; Lee, S. Vertical Si-nanowire n-type tunneling FETs with low subthreshold swing ( $\leq 50 \mathrm{mV} /$ decade) at room temperature. IEEE Electron Device Lett. 2011, 32, 437-439. [CrossRef]

9. Zhao, X.; Heidelberger, C.; Fitzgerald, E.A.; del Alamo, J.A. Source/drain asymmetry in InGaAs vertical nanowire MOSFETs. IEEE Trans. Electron Devices 2017, 64, 2161-2165. [CrossRef]

10. Zheng, P.; Connelly, D.; Ding, F.; Liu, T.K. FinFET evolution toward stacked-nanowire FET for CMOS technology scaling. IEEE Trans. Electron Devices 2015, 62, 3945-3950. [CrossRef]

11. Johansson, S.; Egard, M.; Ghalamestani, S.G.; Borg, B.M.; Berg, M.; Wernersson, L.-E.; Lind, E. RF characterization of vertical InAs nanowire wrap-gate transistors integrated on Si substrates. IEEE Trans. Microw. Theory Tech. 2011, 59, 2733-2738. [CrossRef]

12. Lyu, X.; Chen, X. Vertical power Hk-MOSFET of hexagonal layout. IEEE Trans. Electron Devices 2013, 60, 1709-1715. [CrossRef]

13. Ji, D.; Gupta, C.; Agarwal, A.; Chan, S.H.; Lund, C.; Li, W.; Keller, S.; Mishra, U.K.; Chowdhury, S. Large-area in-situ oxide, GaN interlayer-based vertical trench MOSFET (OG-FET). IEEE Electron Device Lett. 2018, 39, 711-714. [CrossRef]

14. Sharma, R.; Rathore, R.S.; Rana, A.K. Impact of high-k spacer on device performance of nanoscale underlap fully depleted SOI MOSFET. J. Circuits Syst. Comput. 2017, 27, 1850063-1-1850063-13. [CrossRef]

15. Maity, N.P.; Maity, R.; Baishya, S. Ultrathin body partial silicon-on-insulator MOSFET with suppressed floating body effect: A simulation study. J. Nanoelectron. Optoelectron. 2017, 12, 472-479. [CrossRef]

16. Yang, P.; Hook, T.B.; Oldiges, P.J.; Doris, B.B. Vertical slit FET at 7-nm node and beyond. IEEE Trans. Electron Devices 2016, 63, 3327-3334. [CrossRef]

17. Larrieu, G.; Dubois, E. Schottky-barrier source/drain MOSFETs on ultrathin SOI body with a tungsten metallic midgap gate. IEEE Electron Device Lett. 2004, 25, 801-803. [CrossRef]

18. Guan, L.P.; Sin, J.K.O.; Xiong, Z.B.; Liu, H.T. A novel SOI lateral-power MOSFET with a self-aligned drift region. IEEE Electron Device Lett. 2005, 26, 264-266. [CrossRef]

19. Anvarifard, M.K.; Orouji, A.A. Enhanced critical electrical characteristics in a nanoscale low-voltage SOI MOSFET with dual tunnel diode. IEEE Trans. Electron Devices 2015, 62, 1672-1676. [CrossRef]

20. Samal, S.K.; Panth, S.; Samadi, K.; Saeidi, M.; Du, Y.; Lim, S.K. Adaptive regression-based thermal modeling and optimization for monolithic 3-D ICs. IEEE Trans. Comput. Aided Des. Integr. Circuits Syst. 2016, 35, 1707-1720. [CrossRef]

21. Zhang, L.; Song, D.; Xiao, Y.; Lin, X.; Chan, M. On the formulation of self-heating models for circuit simulation. IEEE J. Electron Devices Soc. 2018, 6, 291-297. [CrossRef]

22. Xu, Y.; Ho, C.N.M.; Ghosh, A.; Muthumuni, D. Design, Implementation, and Validation of Electro-Thermal Simulation for SiC MOSFETs in Power Electronic Systems. IEEE Trans. Ind. Appl. 2021, 57, 2714-2725. [CrossRef] 
23. COMSOL Multiphysics. Available online: http://cn.comsol.com/blogs/how-to-perform-a-3d-analysis-of-a-semiconductordevice/ (accessed on 25 April 2021).

24. Saxena, P.; Gorji, N.E. COMSOL simulation of heat distribution in perovskite solar cells: Coupled optical-electrical-thermal 3-D analysis. IEEE J. Photovolt. 2019, 9, 1693-1698. [CrossRef]

25. Jia, Y.; Xiao, F.; Duan, Y.; Luo, Y.; Liu, B.; Huang, Y. PSpice-COMSOL-Based 3-D Electrothermal-Mechanical Modeling of IGBT Power Module. IEEE J. Emerg. Sel. Top. Power Electron. 2020, 8, 4173-4185. [CrossRef]

26. Zhou, H.; Alghmadi, S.; Si, M.; Qiu, G.; Ye, P.D. $\mathrm{Al}_{2} \mathrm{O}_{3} / \beta-\mathrm{Ga}_{2} \mathrm{O}_{3}(-201)$ interface improvement through piranha pretreatment and postdeposition annealing. IEEE Electron Device Lett. 2016, 37, 1411-1414. [CrossRef]

27. Ohara, J.; Kano, K.; Takeuchi, Y.; Ohya, N.; Otsuka, Y.; Akita, S. A new deep reactive ion etching process by dual sidewall protection layer. In Proceedings of the IEEE Thirteenth Annual International Conference on Micro Electro Mechanical Systems (Cat. No.00CH36308), Miyazaki, Japan, 23-27 January 2000; pp. 277-282.

28. Winkler, F.; Killge, S.; Fischer, D.; Richter, K.; Hiess, A.; Bartha, J.W. TSV transistor-Vertical metal gate FET inside a through silicon via. IEEE Electron Device Lett. 2018, 39, 1493-1496. [CrossRef]

29. Memišević, E.; Lind, E.; Wernersson, L.E. Thin electron beam defined hydrogen silsesquioxane spacers for vertical nanowire transistors. J. Vac. Sci. Technol. B 2014, 32, 051211-1-051211-6. [CrossRef]

30. Son, D.; Myeong, I.; Kim, H.; Kang, M.; Jeon, J.; Shin, H. Analysis of electrothermal characteristics of GAA vertical nanoplateshaped FETs. IEEE Trans. Electron Devices 2018, 65, 3061-3064. [CrossRef]

31. Belkhiria, M.; Echouchene, F.; Jaba, N.; Bajahzar, A.; Belmabrouk, H. 2-D-Nonlinear Electrothermal Model for Investigating the Self-Heating Effect in GAAFET Transistors. IEEE Trans. Electron Devices 2021, 68, 954-961. [CrossRef]

32. Fjeldly, T.A.; Shur, M. Threshold voltage modeling and the subthreshold regime of operation of short-channel MOSFETs. IEEE Trans. Electron Devices 1993, 40, 137-145. [CrossRef]

33. Liu, M.; Lentz, F.; Trellenkamp, S.; Hartmann, J.-M.; Knoch, J.; Grutzmacher, D.; Buca, D.; Zhao, Q.-T. Diameter scaling of vertical Ge gate-all-around nanowire pMOSFETs. IEEE Trans. Electron Devices 2020, 67, 2988-2994. [CrossRef]

34. Thingujam, T.; Dai, Q.; Kim, E.; Lee, J.-H. A simulation study on the effects of interface charges and geometry on vertical GAA GaN nanowire MOSFET for low-power application. IEEE Access 2021, 9, 101447-101453. [CrossRef]

35. Vudumula, P.; Kotamraju, S. Design and optimization of SiC super-junction MOSFET using vertical variation doping profile. IEEE Trans. Electron Devices 2019, 66, 1402-1408. [CrossRef]

36. Hsu, P.; Chen, H.; Hwang, T. Stacking Signal TSV for thermal dissipation in global routing for 3-D IC. IEEE Trans. Comput. Aided Des. Integr. Circuits Syst. 2014, 33, 1031-1042.

37. Zhou, W.; Zhong, X.; Sheng, K. High temperature stability and the performance degradation of SiC MOSFETs. IEEE Trans. Power Electron. 2014, 29, 2329-2337. [CrossRef]

38. Chen, T.P.; Huang, J.Y.; Tse, M.S.; Tan, S.S.; Ang, C.H. Gate oxide thickness dependence of edge charge trapping in NMOS transistors caused by charge injection under constant-current stress. IEEE Trans. Electron Devices 2003, 50, 1548-1550. [CrossRef] 